\title{
OPTICAL CYCLE MODELLING FOR RbYb AND CsYb MOLECULES
}

\author{
D. N. Menailava ${ }^{a}$ and M. B. Shundalau ${ }^{a, b^{*}}$
}

UDC 535.34

The probabilities of two-step schemes (optical cycles) for the transition of polar RbYb and CsYb molecules from highly excited vibrational states into the ground vibronic state were calculated. It was shown that the most effective optical cycles involved the excited $2^{2} \Sigma_{1 / 2}^{+}$-state.

Keywords: polar diatomic molecules, RbYb, CsYb, vibronic states, optical cycle.

Introduction. Cold $(1 \mathrm{mK}-1 \mathrm{~K})$ and ultracold $(<1 \mathrm{mK})$ polar diatomic molecules containing different alkali metals are promising species for various basic and applied problems, e.g., verifying the theory of the electron dipole moment, creating quantum computers, etc. [1,2]. Polar diatomic molecules for these purposes must be produced in the ground and so-called absolute rovibronic state $X(v=0, J=0)$. Photoassociation is one method for synthesizing such molecules [1-3]. In it, atoms with a low translation temperature due to preliminary laser cooling form molecules in highly excited (near the dissociation limit) short-lived vibrational states of the ground electronic state with internuclear distances far exceeding the equilibrium value. The weakly associated atomic dimers exist in rotational states with small $J$ values because of the low initial temperature of the atoms. Then, a two-step scheme can be used for the transition of the molecules into the absolute rovibronic state $[4,5]$. In the first step, molecules with low rotational temperatures in weakly associated vibrational states of the ground electronic state $X\left(v^{\prime \prime}, J^{\prime \prime}=0\right)$ absorb a pump laser photon and transition into vibrational-rotational states of an excited electronic state $E\left(v^{\prime}, J^{\prime}=1\right)$ (pump or transition 1 in Fig. 1). The pump laser wavelength can be adjusted to avoid populating rovibronic states $E\left(v^{\prime}, J^{\prime} \neq 1\right)$ that are not involved in the scheme. The second step of the scheme occurs as a result of stimulated radiative transitions of molecules from the excited rovibronic states $E\left(v^{\prime}, J^{\prime}=1\right)$ into an absolute rovibronic state $X\left(v^{\prime \prime}=0, J^{\prime \prime}=0\right)$ (dump or transition 2 in Fig. 1). A pulse from a second laser phase-synchronized with the pump laser is used to produce coherent pumping and stimulated emission and to avoid undesired spontaneous transitions and branching. This stimulated Raman adiabatic passage (STIRAP) method [6] can completely control the population of internal degrees of freedom, which increases practically to $100 \%$ the efficiency of a given optical cycle scheme. A suitable scheme with the maximum probability for the cycle $X\left(v^{\prime \prime}, J^{\prime \prime}=0\right) \rightarrow E\left(v^{\prime}, J^{\prime}=1\right) \rightarrow X\left(v^{\prime \prime}=0, J^{\prime \prime}=0\right)$ must be chosen for high efficiency. In turn, the exact potential-energy functions (PEFs) of the ground and excited molecular states must be known in order to construct and optimize the optical cycles. Such PEFs can be obtained by analyzing high-resolution rovibronic spectra or by using quantum-chemical calculations at a high theory level.

Diatomic molecules containing an alkali metal and ytterbium atom (e.g., $\mathrm{RbYb}$ and $\mathrm{CsYb}$ ) have constant electricdipole and magnetic-dipole moments because they contain an unpaired electron. This provides additional opportunities to control gas molecules cooled to low and ultralow temperatures by using electric and magnetic fields [7, 8].

The term $X^{2} \Sigma^{+}$of the ground electronic state of $\mathrm{RbYb}$ and $\mathrm{CsYb}$ corresponds to the dissociative limit $\mathrm{Rb}(5 s)+$ $\mathrm{Yb}\left(6 s^{2}\right)\left[\mathrm{Cs}(6 s)+\mathrm{Yb}\left(6 s^{2}\right)\right]$ (limit I). Lower excited electronic terms $2^{2} \Sigma^{+}$and $1^{2} \Pi$ arise from electronic configurations $\mathrm{Rb}(5 p)+\mathrm{Yb}\left(6 s^{2}\right)\left[\operatorname{or} \mathrm{Cs}(6 p)+\mathrm{Yb}\left(6 s^{2}\right)\right]$ (dissociative limit II). Terms $X^{2} \Sigma^{+}$and $2^{2} \Sigma^{+}$correspond to states $X^{2} \Sigma_{1 / 2}^{+}$and $2^{2} \Sigma_{1 / 2}^{+}$ for calculating the spin-orbit coupling (SOC). Term $1^{2} \Pi$ is split into $1^{2} \Pi_{3 / 2^{-}}$and $1^{2} \Pi_{1 / 2}$-states. Figure 1 shows the PEFs of $\mathrm{RbYb}$ lower electronic states [9]. The PEFs of $\mathrm{CsYb}$ are analogous [10]. The next nine spin-mixed excited molecular states of $\mathrm{RbYb}$ arise by combining the $\mathrm{Rb}$ ground ${ }^{2} S$ term and the $\mathrm{Yb}$ excited triplet ${ }^{3} \mathrm{P}$ term (dissociative limit III). Six $\mathrm{CsYb}$

${ }^{*}$ To whom correspondence should be addressed.

${ }^{\mathrm{a} B e l a r u s i a n}$ State University, 4 Nezavisimost' Ave., Minsk, 220030, Belarus; e-mail: shundalov@bsu.by; ${ }^{b}$ A. N. Sevchenko Institute of Applied Physical Problems, Belarusian State University, Minsk, Belarus. Translated from Zhurnal Prikladnoi Spektroskopii, Vol. 84, No. 4, pp. 515-520, July-August, 2017. Original article submitted December 15, 2016. 


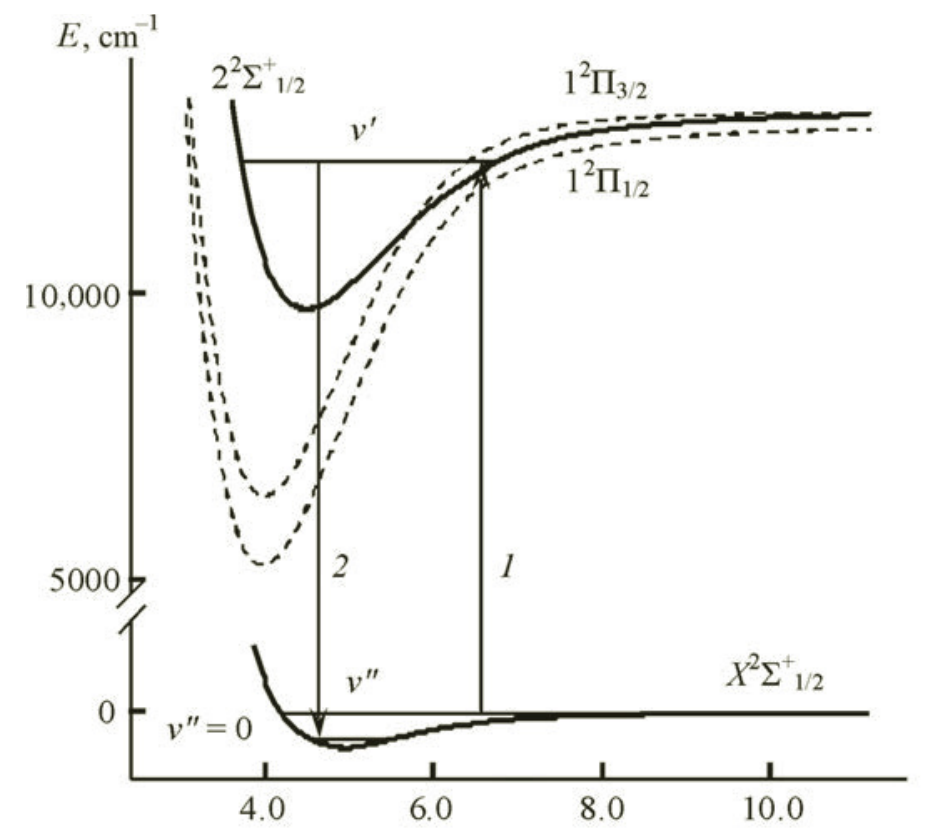

Fig. 1. Optical cycle scheme involving ground $X^{2} \Sigma_{1 / 2}^{+}$and excited $2^{2} \Sigma_{1 / 2}^{+}$-states of RbYb: 1) excitation channel (pump); 2) stimulated transition channel (dump).

molecular spin-mixed states that arise by combining the $\mathrm{Yb}$ ground ${ }^{1} S$ term and Cs excited ${ }^{2} D$ (five states) and ${ }^{2} S$ (one state) terms (dissociative limits IV and V) are situated between dissociative limits II and III.

Electronic states of RbYb were studied experimentally $[3,7]$ and theoretically $[8,11-15]$. Energies of only vibrational states 7 and 15 were determined experimentally near the dissociative limits of the ground $X^{2} \Sigma_{1 / 2}^{+}[3]$ and excited $1^{2} \Pi_{1 / 2}[7]$ electronic states. It is noteworthy that the PEFs of the lower electronic states of $\mathrm{RbYb}$ that were calculated by the different methods differed considerably [8, 11-15]. The PEFs of the ground and several lower excited electronic states of CsYb were also calculated $[12,15]$. The results of these calculations were also inconsistent. The dissociation energy of the ground state according to one calculation [15] was greater than three times that obtained from the other [12].

Herein, the previously calculated $[9,10]$ spectral and energy characteristics of $\mathrm{RbYb}$ and $\mathrm{CsYb}$ molecular vibronic states were used to model optical cycles for the efficient transition of these molecules into the absolute rovibronic states.

Calculations. The overall probability of optical cycle $P$ is proportional to the product of the probability $P_{X \rightarrow E}^{v^{\prime \prime}, J^{\prime \prime} \rightarrow v^{\prime}, J^{\prime}}$ of optical excitation $X\left(v^{\prime \prime}, J^{\prime \prime}\right) \rightarrow E\left(v^{\prime}, J^{\prime}\right)$ and probability $P_{E \rightarrow X}^{v^{\prime}, J^{\prime} \rightarrow v^{\prime \prime}=0, J^{\prime \prime}=0}$ of the transition into the ground state $E\left(v^{\prime}, J^{\prime}\right) \rightarrow$ $X\left(v^{\prime \prime}=0, J^{\prime \prime}=0\right)$ (here, transitions with selection rules $\Delta J= \pm 1$ are considered):

$$
P \sim P_{X \rightarrow E}^{v^{\prime \prime}, J^{\prime \prime} \rightarrow v^{\prime}, J^{\prime}} P_{E \rightarrow X}^{v^{\prime}, J^{\prime} \rightarrow v^{\prime \prime}=0, J^{\prime \prime}=0},
$$

where $P_{E \leftrightarrow X}^{v^{\prime}, J^{\prime} \leftrightarrow v^{\prime \prime}, J^{\prime \prime}} \sim\left|E_{E}^{v^{\prime}, J^{\prime}}-E_{X}^{v^{\prime \prime}}, J^{\prime \prime}\right| S_{E \leftrightarrow X} q^{v^{\prime} v^{\prime \prime}} S^{J^{\prime} J^{\prime \prime}} ; E_{E}^{v^{\prime}, J^{\prime}}$ and $E_{X}^{v^{\prime \prime}, J^{\prime \prime}}$, energies of rovibronic states; $S_{E \leftrightarrow X}$, strength of the electronic transition line; $q^{v^{\prime}, v^{\prime \prime}}$, Franck-Condon factor; and $S^{J^{\prime} J^{\prime \prime}}$, Henley-London factor. The effect of rotational degrees of freedom on the overall probabilities turned out to be insignificant (Henley-London factors equal to unity, energies of rotational sublevels small because of a small rotational constant) and was not examined further because the molecules formed by photoassociation had a very low rotational temperature.

Thus, transition dipole moments, which are proportional to the corresponding line strengths, must be considered first when optimizing the optical cycle. Franck-Condon factors are also important and can differ by several orders of magnitude. Furthermore, the excited-state lifetime, which is related to the existence of several additional channels for spontaneous decay, can also be a significant factor.

The probabilities of vibronic transitions were determined from the PEFs of $\mathrm{RbYb}$ [9] and $\mathrm{CsYb}$ [10] ground and lower excited electronic states that were calculated earlier using CASSCF/XMCQDPT2 multi-reference perturbation theory [16] taking into account SOC [16]. The approximation used by us to solve multi-configuration problems considered static 


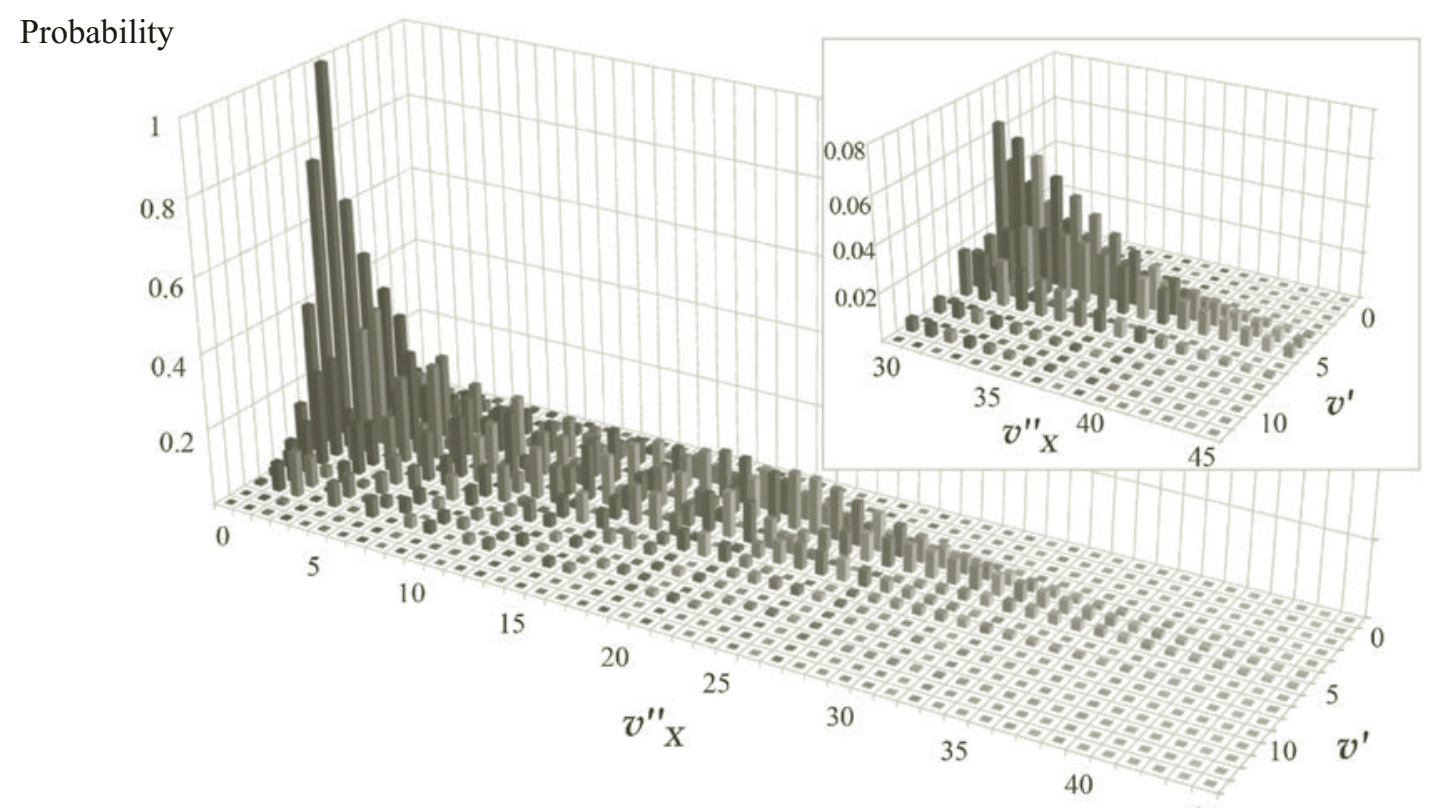

45

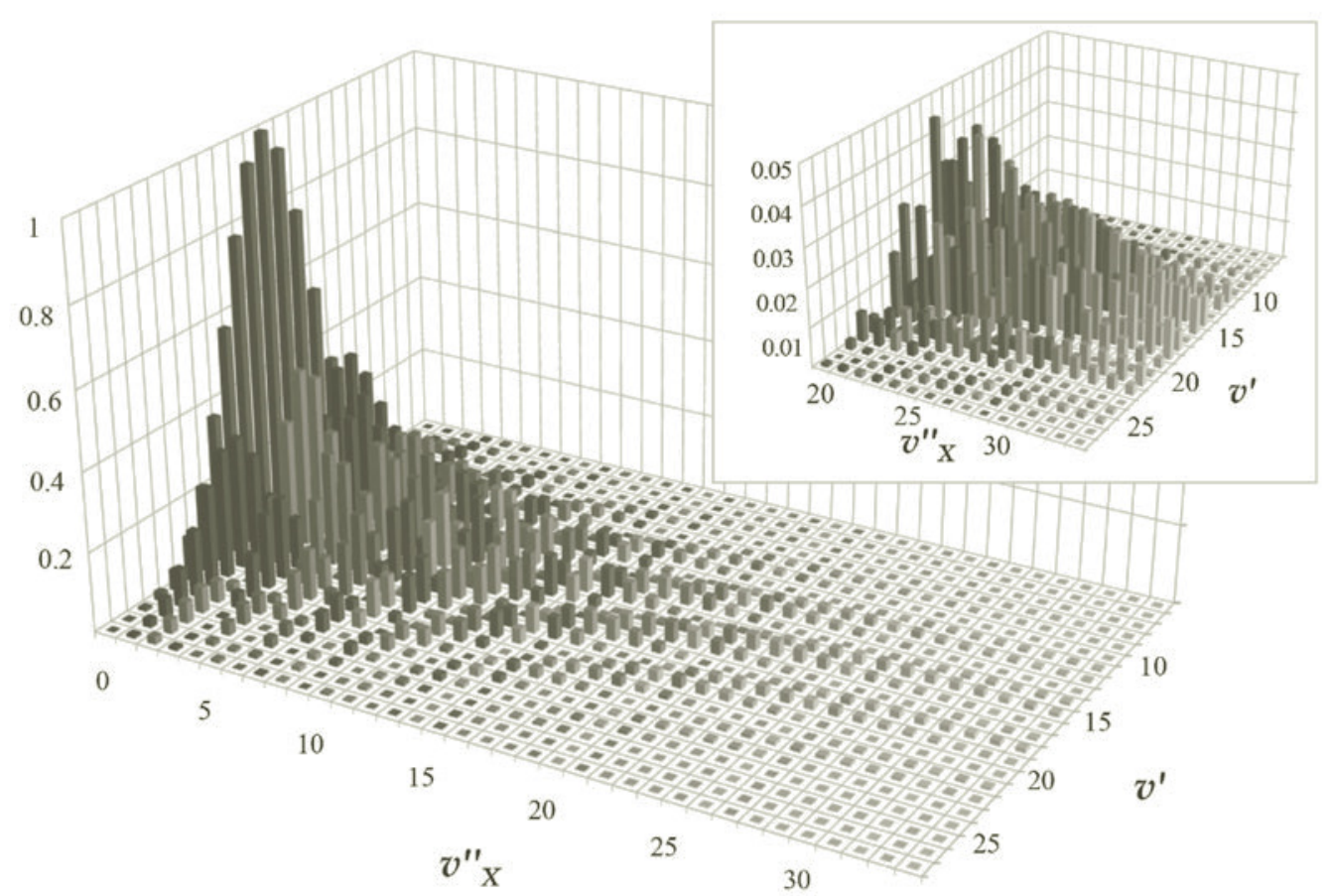

Fig. 2. Probabilities of optical cycles involving the ground $X^{2} \Sigma_{1 / 2^{-}}^{+}$and excited $2^{2} \Sigma_{1 / 2}^{+}$-states of $\mathrm{RbYb}(\mathrm{a})$ and $\mathrm{CsYb}(\mathrm{b})$; in the insets, probabilities of highly excited vibrational states of the ground electronic $X^{2} \Sigma_{1 / 2}^{+}$-state.

(CASSCF) and dynamic (XMCQDPT2) components of the electronic correlation energy. This in combination with relativistic effects (SOC) can highly accurately reproduce [17, 18] experimental [19] energy and spectral characteristics of ground and excited electronic states of both heavy diatomic molecules and complicated organic compounds [20]. In instances where experimental data for the molecular parameters of PEFs of diatomic molecular electronic states are missing (e.g., RbYb and $\mathrm{CsYb}$ ), the agreement between the calculated energies of electronic terms at the dissociative limits and the sums of experimentally determined energies of states of individual atoms can act as an acceptance criterion for the results. The lower electronic states of $\mathrm{RbYb}$ and $\mathrm{CsYb}$ agreed within a few $\mathrm{cm}^{-1}[9,10,21]$. Thus, it was assumed that the calculated PEFs of 

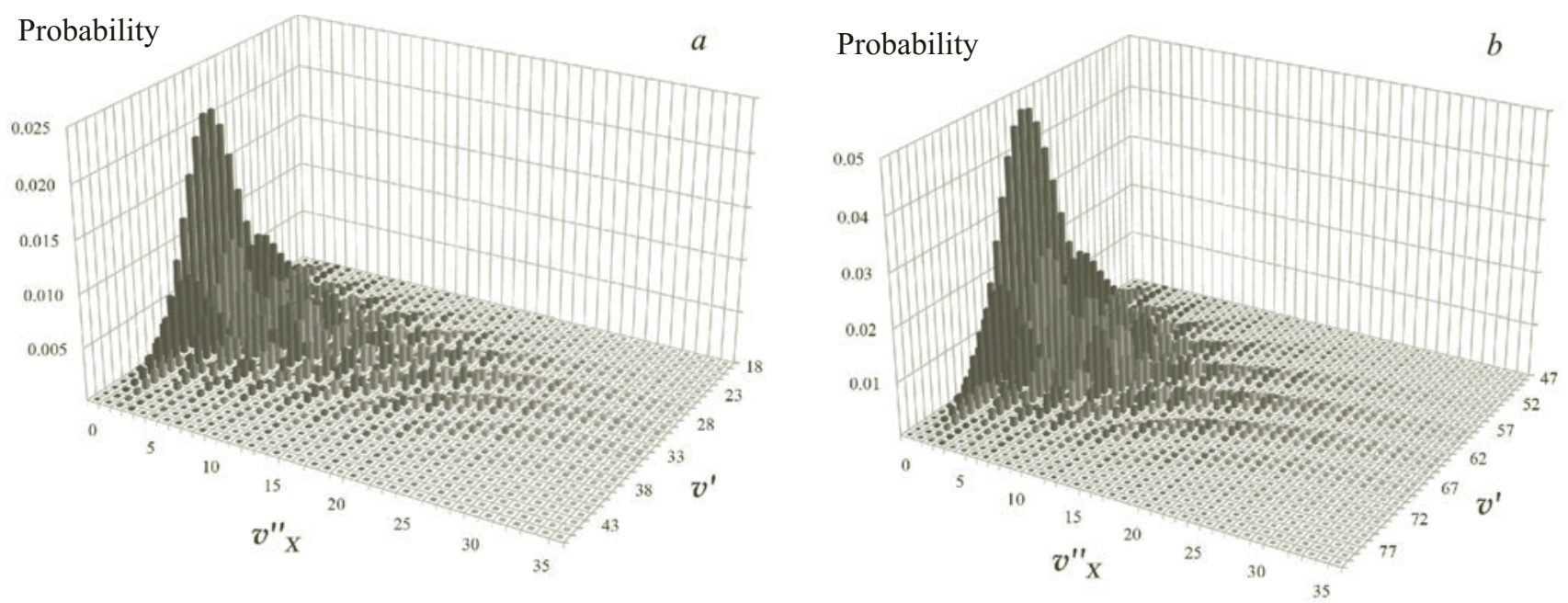

Fig. 3. Probabilities of optical cycles involving the ground $X^{2} \Sigma_{1 / 2}^{+}-$and excited $1^{2} \Pi_{1 / 2}$-states of $\mathrm{RbYb}(\mathrm{a})$ and $\mathrm{CsYb}(\mathrm{b})$.

$\mathrm{RbYb}$ and $\mathrm{CsYb}$ were also close to the experimental values. The PEFs and dipole moments of the electronic transitions were calculated using the Firefly applied quantum-chemistry suite [22]; energies of vibrational states and PEFs, using the LEVEL program [23].

Results and Discussion. Dipole moments of electronic transitions between ground $X^{2} \Sigma_{1 / 2}^{+}$and excited states arising from electronic configurations of atoms at dissociative limits III (for RbYb and CsYb) and IV and V (for CsYb) were different from zero only at small (3.5-8 $\AA$ ) internuclear distances $R$ (Fig. 2 and [9]) because the corresponding transitions at the dissociative limits were forbidden by selection rules for the atoms. Therefore, probabilities $P_{X \rightarrow E}^{v^{\prime \prime} \rightarrow v^{\prime}}$ of optical excitation of the molecules (actually atom pairs with large internuclear distances) from weakly bound vibrational states of the ground electronic term lying near its dissociative limit into vibronic states of molecular terms arising from dissociative limits III, IV, and V were equal to zero near $R>8 \AA$. As a result, optical cycles involving these states turned out to be inefficient. Furthermore, states belonging to dissociative limit II were located between electronic states belonging to limits III, IV, and $\mathrm{V}$ and the $X^{2} \Sigma_{1 / 2}^{+}$ ground state. Therefore, the existence of additional decay channels of excited states had to be considered when constructing optical cycles involving states belonging to limit III. This decreased their average lifetime and reduced the cycle efficiency.

Thus, one of the states belonging to dissociative limit II $\left(2^{2} \Sigma_{1 / 2}^{+}, 1^{2} \Pi_{3 / 2}\right.$, or $\left.1^{2} \Pi_{1 / 2}\right)$ was effectively used as the upper electronic state to construct efficient optical cycles for $\mathrm{RbYb}$ and $\mathrm{CsYb}$.

Figure 2 shows probabilities of the existence of optical cycles for $\mathrm{RbYb}$ and $\mathrm{CsYb}$ involving excited state $2^{2} \Sigma_{1 / 2}^{+}$. The probabilities were calculated for all vibrational states belonging to the $X^{2} \Sigma_{1 / 2^{-}}^{+}$ground and $2^{2} \Sigma_{1 / 2}^{+}$excited electronic states $\left(v^{\prime \prime}=\right.$ $0-45, v^{\prime}=0-125$ for $\mathrm{RbYb}$ and $v^{\prime \prime}=0-36, v^{\prime}=0-119$ for $\left.\mathrm{CsYb}\right)$. The probability distributions were normalized to the maxima corresponding to transitions $v^{\prime \prime}=0 \rightarrow v^{\prime}=6$ for RbYb and $v^{\prime \prime}=0 \rightarrow v^{\prime}=17$ for CsYb. Each $P$ value with coordinates $\left(v^{\prime \prime}, v^{\prime}\right)$ in the diagrams determined the probability of excitation from vibronic state $X^{2} \Sigma_{1 / 2}^{+}\left(v^{\prime \prime}\right)$ into state $2^{2} \Sigma_{1 / 2}^{+}\left(v^{\prime}\right)$ and of radiative transition into the $X^{2} \Sigma_{1 / 2}^{+}\left(v^{\prime \prime}=0\right)$ state.

Figure 2 indicates that optical cycles for states with $v^{\prime \prime}=0-10$ were most efficient. Nevertheless, these excitation channels and stimulated emission of excited vibronic states were not suitable for photoassociation of diatomic molecules because the resulting molecules existed in highly excited vibrational states. Probabilities of optical cycles for these states were units of percent of the maxima (Fig. 2, insets). Optical cycles were most efficient for $v^{\prime \prime}=30-45, v^{\prime}=4-8$ for RbYb and $v^{\prime \prime}=19-36, v^{\prime}=13-23$ for $\mathrm{CsYb}$ for weakly bound vibrational states lying near the dissociative limit.

Figures 3 and 4 show analogous optical cycle schemes involving upper electronic $1^{2} \Pi_{3 / 2}$ and $1^{2} \Pi_{1 / 2}$-states. Here, the probabilities were also normalized to the corresponding maxima for cycles involving the $2^{2} \Sigma_{1 / 2}^{+}$state. Probabilities were calculated for all vibrational states belonging to the ground and excited $\Pi$-states: $v^{\prime}=0-142$ for $1^{2} \Pi_{3 / 2}, v^{\prime}=0-167$ for $1^{2} \Pi_{1 / 2}$ $(\mathrm{RbYb}) ; v^{\prime}=0-137$ for $1^{2} \Pi_{3 / 2}, v^{\prime}=0-144$ for $1^{2} \Pi_{1 / 2}(\mathrm{CsYb})$.

Figures 3 and 4 show that optical cycles involving excited $\Pi$-states were considerably less efficient. The probabilities for optical cycles involving $2^{2} \Sigma_{1 / 2}^{+}$and $\Pi$-states differed primarily by the dipole moments of the corresponding electronic transitions. 

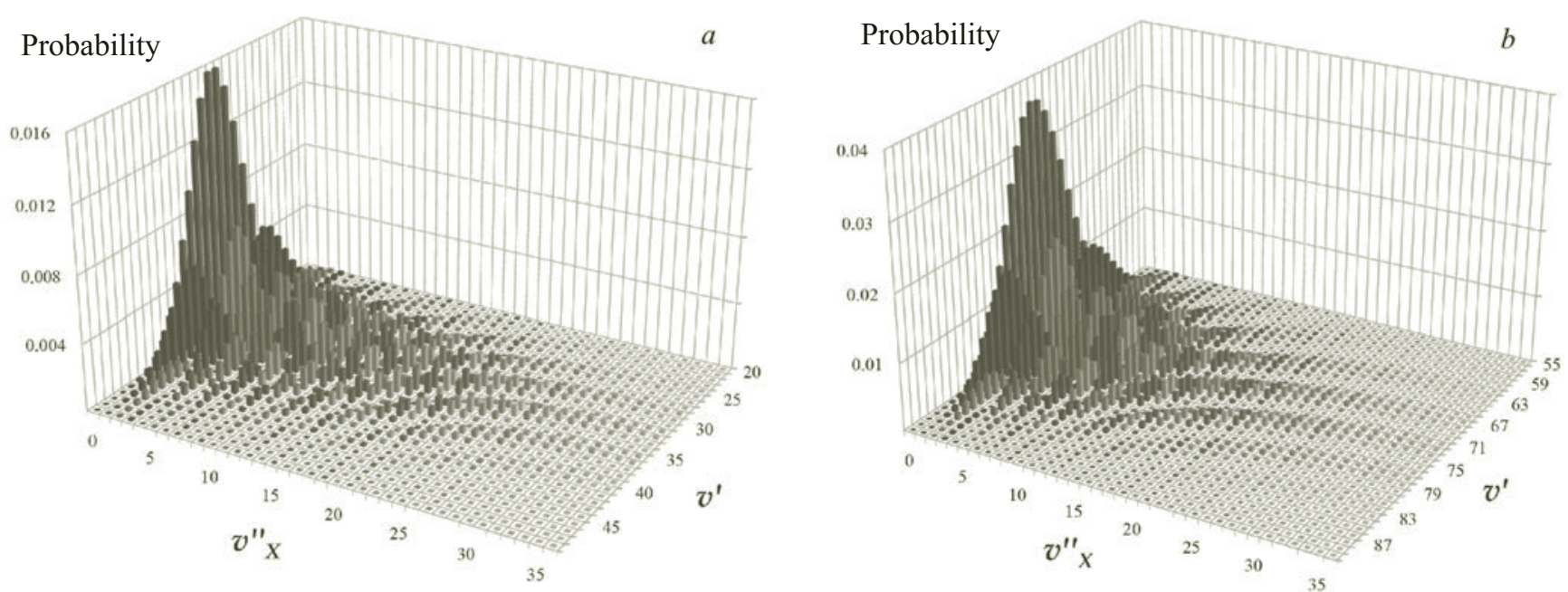

Fig. 4. Probabilities of optical cycles involving the ground $X^{2} \Sigma_{1 / 2}^{+}-$and excited $1^{2} \Pi_{1 / 2}$-states of $\mathrm{RbYb}(\mathrm{a})$ and $\mathrm{CsYb}(\mathrm{b})$.

The average transition dipole moments of the $X^{2} \Sigma_{1 / 2}^{+} \rightarrow 2^{2} \Sigma_{1 / 2}^{+}$transition for RbYb and CsYb were $\sim 8.5$ and $9.2 \mathrm{D}$ over a broad range (3.5-20 $\AA$ ) of internuclear distances whereas those for the $X^{2} \Sigma_{1 / 2}^{+} \rightarrow 1^{2} \Pi_{3 / 2}$ and $X^{2} \Sigma_{1 / 2}^{+} \rightarrow 1^{2} \Pi_{1 / 2}$ transitions decreased from 8-9 D for $R=15-20 \AA$ to $2 \mathrm{D}$ near $R=4 \AA[9,10]$. However, the difference in the Franck - Condon factors, which were 1-2 orders of magnitude less for transitions from the $\Pi$-states than from the $2^{2} \Sigma_{1 / 2}^{+}$-state, played the decisive role.

Conclusions. Probabilities of optical cycles for efficient transition of polar diatomic $\mathrm{RbYb}$ and $\mathrm{CsYb}$ into an absolute rovibronic state involving lower excited electronic states were analyzed. It was shown that two-step schemes through the following channels were most efficient for transitioning $\mathrm{RbYb}$ and $\mathrm{CsYb}$ molecules formed by photoassociation from highly excited vibrational states into the ground state: $X^{2} \Sigma_{1 / 2}^{+}\left(v^{\prime \prime}=30-45\right) \rightarrow 2^{2} \Sigma_{1 / 2}^{+}\left(v^{\prime}=4-8\right) \rightarrow X^{2} \Sigma_{1 / 2}^{+}\left(v^{\prime \prime}=0\right)$ for RbYb and $X^{2} \Sigma_{1 / 2}^{+}$ $\left(v^{\prime \prime}=19-36\right) \rightarrow 2^{2} \Sigma_{1 / 2}^{+}\left(v^{\prime}=13-23\right) \rightarrow X^{2} \Sigma_{1 / 2}^{+}\left(v^{\prime \prime}=0\right)$ for $\mathrm{CsYb}$.

Acknowledgments. The work was supported financially by the State Committee for Science and Technology, Republic of Belarus (Project No. F14LAT-060). We thank Prof. R. Ferber (Laser Center, Latvian University, Riga, Latvia) for useful discussions and recommendations.

\section{REFERENCES}

1. O. Dulieu and C. Gabbanini, Rep. Prog. Phys., 72, 086401 (2009).

2. J. Ulmanis, J. Deiglmayr, M. Repp, R. Wester, and M. Weidemuller, Chem. Rev., 112, 4890-4927 (2012).

3. F. Munchow, C. Bruni, M. Madalinski, and A. Gorlitz, Phys. Chem. Chem. Phys., 13, 18734-18737 (2011).

4. I. Klincare, O. Nikolayeva, M. Tamanis, R. Ferber, E. A. Pazyuk, and A.V. Stolyarov, Phys. Rev. A: At., Mol., Opt. Phys., 85, 062520 (2012).

5. E. A. Pazyuk, A. V. Zaitsevskii, A. V. Stolyarov, M. Tamanis, and R. Ferber, Usp. Khim., 84, 1001-1020 (2015).

6. K. Bergmann, H. Theuer, and B. W. Shore, Rev. Mod. Phys., 70, 1003-1025 (1998).

7. N. Nemitz, F. Baumer, F. Munchow, S. Tassy, and A. Gorlitz, Phys. Rev. A: At., Mol., Opt. Phys., 79, 061403 (2009).

8. S. Kotochigova, A. Petrov, M. Linnik, J. Klos, and P. S. Julienne, J. Chem. Phys., 135, 164108 (2011).

9. M. B. Shundalau and A. A. Minko, Comput. Theor. Chem., 1103, 11-16 (2017).

10. D. N. Meniailava and M. B. Shundalau, Comput. Theor. Chem., 1111, 20-26 (2017).

11. L. K. Sorensen, S. Knecht, T. Fleig, and C. M. Marian, J. Phys. Chem. A, 113, 12607-12614 (2009).

12. E. R. Meyer and J. L. Bohn, Phys. Rev. A: At., Mol., Opt. Phys., 80, 042508 (2009).

13. S. N. Tohme and M. Korek, Chem. Phys., 410, 37-44 (2013).

14. M. Borkowski, P.S. Zuchowski, R. Ciurylo, P.S. Julienne, D. Kedziera, L. Mentel, P. Tecmer, F. Munchow, C. Bruni, and A. Gorlitz, Phys. Rev. A: At., Mol., Opt. Phys., 88, 052708 (2013).

15. D. A. Brue and J. M. Hutson, Phys. Rev. A: At., Mol., Opt. Phys., 87, 052709 (2013). 
16. A. A. Granovsky, J. Chem. Phys., 134, 214113 (2011).

17. M. B. Shundalov and A. A. Min'ko, Zh. Prikl. Spektrosk., 82, 821-825 (2015) [M. B. Shundalau and A. A. Minko, J. Appl. Spectrosc., 82, 901-904 (2015)].

18. M. B. Shundalau, G. A. Pitsevich, A. E. Malevich, A. V. Hlinisty, A. A. Minko, R. Ferber, and M. Tamanis, Comput. Theor. Chem., 1089, 35-42 (2016).

19. A. Pashov, O. Docenko, M. Tamanis, R. Ferber, H. Knockel, and E. Tiemann, Phys. Rev. A: At., Mol., Opt. Phys., 76, 022511 (2007).

20. A. M. Andrianov, I. A. Kashyn, V. M. Andrianov, M. B. Shundalau, A. V. Hlinisty, S. V. Gaponenko, E. V. ShabunyaKlyachkovskaya, A. Matsukovich, A.-M. S. Al-Tamimi, and A. A. El-Emam, J. Chem. Sci., 128, 1933-1942 (2016).

21. NIST Atomic Spectra Database; http://www.nist.gov/pml/data/asd.cfm

22. A. A. Granovsky, Firefly version 8; http://classic.chem.msu.su/gran/firefly/index.html.

23. R. J. Le Roy, Level 8.0: A computer program for solving the radial Schroedinger equation for bound and quasibound levels, University of Waterloo Chemical Physics Research Report CP-663 (2007). 